\title{
Pairing in a two-component ultracold Fermi gas: Phases with broken-space symmetries
}

\author{
Armen Sedrakian, ${ }^{1}$ Jordi Mur-Petit, ${ }^{2}$ Artur Polls, ${ }^{2}$ and Herbert Müther ${ }^{1}$ \\ ${ }^{1}$ Institute for Theoretical Physics, Tübingen University, D-72076 Tübingen, Germany \\ ${ }^{2}$ Departament d'Estructura i Constituents de la Matèria, Universitat de Barcelona, E-08028 Barcelona, Spain
}

(Received 9 February 2005; published 22 July 2005)

\begin{abstract}
We explore the phase diagram of a two-component ultracold atomic Fermi gas interacting with zero-range forces in the limit of weak coupling. We focus on the dependence of the pairing gap and the free energy on the variations in the number densities of the two species while the total density of the system is held fixed. As the density asymmetry is increased, the system exhibits a transition from a homogenous Bardeen-CooperSchrieffer (BCS) phase to phases with spontaneously broken global space symmetries. One such realization is the deformed Fermi surface superfluidity (DFS) which exploits the possibility of deforming the Fermi surfaces of the species into ellipsoidal form at zero total momentum of Cooper pairs. The critical asymmetries at which the transition from DFS to the unpaired state occurs are larger than those for the BCS phase. In this precritical region the DFS phase lowers the pairing energy of the asymmetric BCS state. We compare quantitatively the DFS phase to another realization of superconducting phases with broken translational symmetry: the singleplane-wave Larkin-Ovchinnikov-Fulde-Ferrell phase, which is characterized by a nonvanishing center-of-mass momentum of the Cooper pairs. The possibility of the detection of the DFS phase in the time-of-flight experiments is discussed and quantified for the case of ${ }^{6} \mathrm{Li}$ atoms trapped in two different hyperfine states.
\end{abstract}

DOI: $10.1103 /$ PhysRevA.72.013613

PACS number(s): 03.75.Hh, 03.75.Ss, 74.20.Fg

\section{INTRODUCTION}

The progress achieved in recent years in trapping and manipulating ultracold fermion gases has focused much theoretical attention on the pairing properties of dilute fermionic systems. Current experiments with ${ }^{6} \mathrm{Li}$ and ${ }^{40} \mathrm{~K}$ atoms are carried out at temperatures which are a fraction $(\sim 0.1-0.3)$ of the Fermi temperature [1-8]. These systems are thus characterized by a filled Fermi sea, and at sufficiently low temperatures attractive two-body interactions are expected to drive the Cooper pairing instability $[9,10]$. The strength of the two-body interactions can be tuned using the Feshbach resonance mechanism by varying the external magnetic field [11-13]; thus, the entire range from weak to strong couplings can be probed. In the crossover region the Feshbach resonance may strongly enhance the pairing interaction and give rise to high-temperature superfluidity [14-25]. Recent experiments have probed the condensation of fermionic pairs above the Feshbach resonance, where the system does not support a genuine two-body bound state [26-28]. The nature of these pairs (tightly bound molecules versus extended Cooper pairs) is not clear yet, and the signatures of the superfluid phase transition, which are manifest in the collective and hydrodynamic behavior of these systems [31,32], have been searched for. The measured collective modes of ${ }^{6} \mathrm{Li}$ were found to be consistent with superfluid hydrodynamics and provide some evidence for superfluidity in a resonantly interacting Fermi gas [28]. A gap in the quasiparticle spectrum of a two-component gas of ${ }^{6} \mathrm{Li}$ was recently observed using radio-frequency spectroscopy [33].

The $s$-wave interaction dominates the pairing interaction in cases where (i) the pairing is among atoms of equal mass number but belonging to different hyperfine states or (ii) the pairing is between different species (in mixtures of atomse.g., ${ }^{6} \mathrm{Li}$ and ${ }^{40} \mathrm{~K}$ ), in which case the symmetric BCS limit is not realized [34]. Systems where two hyperfine levels are populated have been created and studied experimentally with ${ }^{6} \mathrm{Li}$ and ${ }^{40} \mathrm{~K}$ atoms [1-8,26-30]. These systems are characterized by a hierarchy of scales: the typical range of the van der Waals forces is $R \leqslant 10^{-6} \mathrm{~cm}$ while the de Broglie wavelength of particles at the top of the Fermi sea is $k_{F}$ $\sim 10^{3}-10^{4} \mathrm{~cm}^{-1}$. Since $k_{F} R \ll 1$, the interaction can be approximated by a zero-range force which is characterized by the $s$-wave scattering length $a_{S}$. We specify our discussion below to the case where two hyperfine states of ${ }^{6} \mathrm{Li}$ are populated, in which case the scattering length in units of the Bohr radius is $a_{S} / a_{B}=-2160$. For typical values of Fermi momenta quoted above $k_{F} a_{S} \simeq 0.04$ and the system is in the weak-coupling regime, since $\nu\left(k_{F}\right) U_{0}=(2 / \pi) k_{F}\left|a_{S}\right| \ll 1$, where $\nu\left(k_{F}\right)=m k_{F} /\left(2 \pi^{2} \hbar^{2}\right)$ is the density of states at the Fermi surface and $U_{0}=4 \pi \hbar^{2} a_{S} / m$ is the strength of the contact interaction. For larger values of $k_{F} a_{S} \sim 1$ the weight of the negative-energy states to the single-particle spectral function is not negligible - the bound states need to be incorporated in the theory along with the pair correlations on the same footing [35].

The BCS theory predicts a suppression of the pairing correlations when the Fermi energies or, equivalently, the densities of the two hyperfine states $|1\rangle$ and $|2\rangle$, denoted below as $\rho_{\uparrow / \downarrow}$, are different. In the low-density and weak-coupling limit $\left(k_{F}\left|a_{S}\right| \ll 1\right)$ the value of the critical density asymmetry $\alpha=\left(\rho_{\uparrow}-\rho_{\downarrow}\right) /\left(\rho_{\uparrow}+\rho_{\downarrow}\right)$, for which the superfluidity vanishes, follows from the relation $[36,37]$

$$
\frac{\Delta(\alpha)}{\Delta_{0}}=\sqrt{1-\frac{4 \alpha}{3} \frac{\mu}{\Delta_{0}}},
$$

where $\Delta_{0} \simeq 8 e^{-2} \mu \exp \left[-\pi /\left(2 k_{F}\left|a_{S}\right|\right)\right]$ is the gap in the symmetric system and $\mu$ is the chemical potential. Therefore, the gap disappears for asymmetries $\alpha>\alpha_{\mathrm{c}}^{B C S}=3 \Delta_{0} /(4 \mu)$. For ex- 
ample, if the pairing is between ${ }^{6} \mathrm{Li}$ atoms in the states $|1\rangle$ $=\left|F=3 / 2, m_{F}=3 / 2\right\rangle$, and $|2\rangle=|3 / 2,1 / 2\rangle$, in which case the pairing interaction is characterized by a scattering length $a_{S} / a_{B}=-2160$, the maximum asymmetry at which BCS pairing is possible is $\alpha_{\mathrm{c}}^{B C S} \simeq 0.055$ for a density $\rho=\rho_{\uparrow}+\rho_{\downarrow}=3.8$ $\times 10^{12} \mathrm{~cm}^{-3}$ (corresponding to $k_{F}\left|a_{S}\right|=0.55$ ).

One purpose of this work is to show that the pairing correlations in ultracold atomic gases can persist for density asymmetries $\alpha>\alpha_{\mathrm{c}}^{B C S}$ and can be enhanced for $\alpha<\alpha_{\mathrm{c}}^{B C S}$ if the Fermi spheres of two hyperfine states are deformed into ellipsoids in momentum space. In the strong-coupling regime $\left(\Delta_{0} / \mu \sim 0.3\right)$ the superconducting state featuring deformed Fermi surfaces was found preferable to the spherically symmetric BCS state [38]. Here we rather explore the weakcoupling regime. Alternatively, the homogeneous BCS phase can evolve into the Larkin-Ovchinnikov-Fulde-Ferrell (LOFF) phase [39,40], which again sustains $\alpha>\alpha_{\mathrm{c}}^{B C S}$ asymmetries by allowing the Cooper pairs to carry finite centerof-mass momentum (the Fermi surfaces in the LOFF phase are spherical).

There has been much interest in the LOFF phase outside the condensed matter context in connection with hadronic systems under extreme conditions where the interactions are mediated by the strong force (see Ref. [46]), but no experimental signature(s) of its realization has been proposed so far. Atomic systems offer a novel setting for studying the LOFF phase under conditions that are more favorable than those in solids (absence of the lattice effects, access to the momentum distribution in the system through time-of-flight experiments) [41-45]. Atomic systems also offer the possibility of novel realizations of the LOFF phase which, for example, invoke $P$-wave anisotropic interactions [41] or finite-size systems $[44,45]$.

While the LOFF and DFS phases break global space symmetries, the way they do so is fundamentally different: the LOFF phase breaks both the rotational and translational symmetries due to the finite momentum of the condensate and irrespective of the form of the lattice structure; the DFS phase breaks only the rotational symmetry from $\mathrm{O}(3)$ down to $\mathrm{O}(2)$. We shall compare below the realizations of the DFS and LOFF phases in the ${ }^{6} \mathrm{Li}$ gas assuming that the order parameter in the LOFF phase has a simple plane-wave form; in addition, we shall describe an experimental signature of the DFS phase that can be established in time-of-flight experiments and that would allow one to distinguish the DFS phase from the competing phases. A brief account of this argument was given earlier in Ref. [47]. Yet another possible alternative is the phase separation of the superconducting and normal phases in real space, such that the superconducting phase contains particles with the same chemical potentials-i.e., is symmetric - while the normal phase remains asymmetric; see Refs. [48-50]. A comparison of the heterogeneous phase with the alternatives would require knowledge of the poorly known surface tension between superconducting and normal phases, and will not be attempted here.

This paper is organized as follows. Starting from Dyson's equations, we derive in Sec. II the dispersion relations of elementary excitations in an asymmetrical superfluid. Section III discusses the gap equation and its regularization for contact interactions. Section IV is devoted to modifications of the single-particle spectra needed to describe the LOFF and DFS phases. Numerical results for the case of ${ }^{6} \mathrm{Li}$ gas are presented in Sec. V. Section VI discusses the possibility of observing the DFS phase in time-of-flight experiments.

\section{FORMALISM}

Consider a uniform gas of fermionic atoms with two hyperfine states, which we assign labels $\uparrow$ and $\downarrow$ (these states equivalently can be thought of as pseudospins.). The model Hamiltonian that describes our system is

$$
\begin{aligned}
\hat{H}= & \frac{1}{2 m} \sum_{\alpha} \int d^{3} x \nabla \hat{\psi}_{\alpha}^{\dagger}(\boldsymbol{x}) \nabla \hat{\psi}_{\alpha}(\boldsymbol{x}) \\
& -\sum_{\alpha \beta} \int d^{3} x \int d^{3} x^{\prime} \hat{\psi}_{\alpha}^{\dagger}(\boldsymbol{x}) \hat{\psi}_{\beta}^{\dagger}\left(\boldsymbol{x}^{\prime}\right) V\left(\boldsymbol{x}, \boldsymbol{x}^{\prime}\right) \hat{\psi}_{\beta}\left(\boldsymbol{x}^{\prime}\right) \hat{\psi}_{\alpha}(\boldsymbol{x}),
\end{aligned}
$$

where $\hat{\psi}_{\alpha}^{\dagger}(\boldsymbol{x})$ and $\hat{\psi}_{\alpha}(\boldsymbol{x})$ are the creation and annihilation operators of a state at the space point specified by the position vector $\boldsymbol{x}$ and pseudospin $\alpha(=\uparrow, \downarrow), m$ is the atom's bare mass, and $V\left(\boldsymbol{x}, \boldsymbol{x}^{\prime}\right)$ is the two-body potential (here and below the volume $\Omega=1$ ). The one-body propagator in the superfluid state is a $2 \times 2$ matrix in Gor'kov space,

$$
\begin{aligned}
G\left(x, x^{\prime}\right) & =\left(\begin{array}{cc}
G_{\alpha \beta}\left(x, x^{\prime}\right) & F_{\alpha \beta}\left(x, x^{\prime}\right) \\
-F_{\alpha \beta}^{\dagger}\left(x, x^{\prime}\right) & \bar{G}_{\alpha \beta}\left(x, x^{\prime}\right)
\end{array}\right) \\
& =\left(\begin{array}{cc}
-i\left\langle T \psi_{\alpha}(x) \psi_{\beta}^{\dagger}\left(x^{\prime}\right)\right\rangle & \left\langle\psi_{\alpha}(x) \psi_{\beta}\left(x^{\prime}\right)\right\rangle \\
\left\langle\psi_{\alpha}^{\dagger}(x) \psi_{\beta}^{\dagger}\left(x^{\prime}\right)\right\rangle & -i\left\langle\widetilde{T} \psi_{\alpha}(x) \psi_{\beta}^{\dagger}\left(x^{\prime}\right)\right\rangle
\end{array}\right),
\end{aligned}
$$

where $x=(\boldsymbol{x}, t)$, the Greek indices stand for the pseudospin, $T$ and $\tilde{T}$ are the time-ordering and time-anti ordering symbols, and $G_{\alpha \beta}\left(x, x^{\prime}\right)$ and $F_{\alpha \beta}^{\dagger}\left(x, x^{\prime}\right)$ are the normal and anomalous propagators [51]. The propagators above are assumed to be ordered on the Schwinger-Keldysh real-time contour [52], which permits a finite-temperature treatment of the problem. To keep our presentation concise, we shall not write down the explicit form of the correlation functions on the contour; our final expressions are written for retarded correlation functions. The $2 \times 2$ matrix Green's function satisfies the familiar Dyson equation

$$
\begin{aligned}
\underline{G}_{\alpha \beta}\left(x, x^{\prime}\right)= & \underline{G}_{\alpha \beta}^{0}\left(x, x^{\prime}\right)+\sum_{\gamma, \delta} \int d^{4} x^{\prime \prime} d^{4} x^{\prime \prime \prime} \underline{G}_{\alpha \gamma}^{0}\left(x, x^{\prime \prime \prime}\right) \\
& \times \sum_{\gamma \delta}\left(x^{\prime \prime \prime}, x^{\prime \prime}\right) \underline{G}_{\delta \beta}\left(x^{\prime \prime}, x^{\prime}\right),
\end{aligned}
$$

where the free propagators $G_{\alpha \beta}^{0}\left(x, x^{\prime}\right)$ are diagonal in Gor'kov space; the underline indicates that the propagators and self-energies are matrices in this space. The components of the Fourier transform of Eq. (4) with respect to the difference of the space arguments of the two-point correlation functions are

$$
G_{\alpha \beta}(p)=G_{0 \alpha \beta}(p)+G_{0 \alpha \gamma}(p)\left[\Sigma_{\gamma \delta}(p) G_{\delta \beta}(p)+\Delta_{\gamma \delta}(p) F_{\delta \beta}^{\dagger}(p)\right],
$$




$$
F_{\alpha \beta}^{\dagger}(p)=G_{0 \alpha \gamma}(-p)\left[\Delta_{\gamma \delta}^{\dagger}(p) G_{\delta \beta}(p)+\Sigma_{\gamma \delta}(-p) F_{\delta \beta}^{\dagger}(p)\right],
$$

where $p$ is the four-momentum, $G_{0 \alpha \beta}(p)$ is the free normal propagator, and $\Sigma_{\alpha \beta}(p)$ and $\Delta_{\alpha \beta}(p)$ are the normal and anomalous self-energies; summation over repeated indices is understood. The Dyson equations for the components $\bar{G}_{\alpha \beta}(p)$ and $F_{\alpha \beta}(p)$ follow from Eqs. (5) and (6) through the timereversal operation.

Below, we shall assume that the interactions conserve spin-i.e., $\quad G_{\alpha \beta}(p)=\delta_{\alpha \beta} G(p)$ and $\Sigma_{\alpha \beta}(p)=\delta_{\alpha \beta} \Sigma(p)$-and concentrate on the pairing in the state of zero total spin and orbital angular momentum $S=L=0$. Thus the anomalous propagators and self-energies must be antisymmetric with respect to the spin indices,

$$
F_{\alpha \beta}^{\dagger}(p)=g_{\alpha \beta} F^{\dagger}(p), \quad \Delta_{\alpha \beta}^{\dagger}(p)=g_{\alpha \beta} \Delta^{\dagger}(p),
$$

where $g_{\alpha \beta} \equiv i \sigma_{y}$ is the spin matrix, with $\sigma_{y}$ being the second component of the vector of Pauli matrices. It is convenient to rewrite Eqs. (5) and (6) in terms of auxiliary Green's functions, which describe the unpaired state of the system:

$$
G_{\alpha \beta}^{N}(p)=G_{0 \alpha \beta}(p)+G_{\alpha \gamma}^{N}(p) \Sigma_{\gamma \delta}(p) G_{0 \delta \beta}^{N}(p) .
$$

The formal solution of Eq. (8) for the retarded propagators in terms of the self-energy $\Sigma(p)$ is

$$
\begin{aligned}
G_{\alpha \beta}^{N}( \pm p) & =\delta_{\alpha \beta}\left[ \pm \omega-\xi_{p \sigma}-\Sigma_{\sigma}( \pm p)\right]^{-1} \\
& =\delta_{\alpha \beta}\left[ \pm(\omega+i \eta)-\varepsilon_{p \sigma}\right]^{-1}+O\left(\operatorname{Im} \Sigma_{\sigma}( \pm p)\right),
\end{aligned}
$$

where $\xi_{p \sigma}=p^{2} / 2 m_{\sigma}-\mu_{\sigma}$ is the energy of a spin- $\sigma$ free particle relative to the chemical potential $\mu_{\sigma}$; the second line follows in the quasiparticle approximation, which keeps the leading-order term in the expansion of the propagator with respect to the small imaginary part of the self-energy. The quasiparticle dispersion relation in the normal state is then given by $\varepsilon_{p \sigma}=\xi_{p \sigma}+\operatorname{Re} \Sigma_{\sigma}( \pm p)$. Combining Eqs. (5) and (6) with Eq. (8) one finds

$$
\begin{gathered}
G_{\alpha \beta}(p)=G_{\alpha \gamma}^{N}(p)\left[\delta_{\gamma \beta}+\Delta_{\gamma \delta}(p) F_{\delta \beta}^{\dagger}(p)\right], \\
F_{\alpha \beta}^{\dagger}(p)=G_{\alpha \gamma}^{N}(-p) \Delta_{\gamma \delta}^{\dagger}(p) G_{\delta \beta}(p) ;
\end{gathered}
$$

these equations are easily solved to obtain the propagators

$$
\begin{gathered}
G_{\uparrow \downarrow}(p)=\frac{\omega+E_{S} \pm E_{A}}{\left(\omega-E_{A}\right)^{2}-E_{S}^{2}-\Delta^{2}}, \\
F^{\dagger}(p)=-\frac{\Delta^{\dagger}}{\left(\omega-E_{A}\right)^{2}-E_{S}^{2}-\Delta^{2}},
\end{gathered}
$$

where $E_{S}=\left(\varepsilon_{p \uparrow}+\varepsilon_{p \downarrow}\right) / 2$ and $E_{A}=\left(\varepsilon_{p \uparrow}-\varepsilon_{p \downarrow}\right) / 2$ are, respectively, the parts of the spectrum which are symmetric and antisymmetric under time-reversal operation. The poles of these propagators (which must be identical) define the dispersion relation of the quasiparticles in the paired state:

$$
\omega_{1 / 2}=E_{A} \pm \sqrt{E_{S}^{2}+\Delta^{2}} .
$$

Note that the quasiparticle spectrum is twofold split due to the asymmetry in the number of the spin-up and spin-down atoms. In the symmetric limit $\left(E_{A}=0\right)$ we recover the ordinary BCS dispersion relation.

\section{GAP EQUATION}

To obtain a closed set of equations we need to specify the approximation to the anomalous self-energy. In the meanfield (BCS) approximation,

$$
\begin{aligned}
\Delta^{\dagger}(p) & =i \int V\left(\boldsymbol{p}, \boldsymbol{p}^{\prime}\right) F^{\dagger}\left(p^{\prime}\right) \frac{d^{4} p^{\prime}}{(2 \pi)^{4}} \\
& =i \int V\left(\boldsymbol{p}, \boldsymbol{p}^{\prime}\right) G_{\downarrow}^{N}\left(-p^{\prime}\right) \Delta^{\dagger}\left(p^{\prime}\right) G_{\uparrow}\left(p^{\prime}\right) \frac{d^{4} p^{\prime}}{(2 \pi)^{4}},
\end{aligned}
$$

where $V\left(\boldsymbol{p}, \boldsymbol{p}^{\prime}\right)$ is the Fourier transform of the two-body interaction $V\left(\boldsymbol{x}, \boldsymbol{x}^{\prime}\right)$ which is responsible for the pairing. The pairing interaction can be renormalized in such a way that integration in Eq. (15) is restricted to the vicinity of the Fermi surface. This permits us to approximate smooth functions of momentum by their value at the Fermi momentum. We introduce a momentum renormalization scale $\Lambda$ such that $\Delta \ll \varepsilon_{\Lambda} \ll \min \left[\varepsilon_{F \uparrow}, \varepsilon_{F \downarrow}\right]$, where $\varepsilon_{F \uparrow \downarrow}$ are the Fermi energies of spin-up and down species. Then,

$$
\Delta^{\dagger}(p)=i \int U\left(\boldsymbol{p}, \boldsymbol{p}^{\prime}\right) G_{\downarrow}^{N}\left(-p^{\prime}\right) \Delta^{\dagger}\left(p^{\prime}\right) G_{\uparrow}\left(p^{\prime}\right) \theta\left(\Lambda-\left|\boldsymbol{p}^{\prime}\right|\right) \frac{d^{4} p^{\prime}}{(2 \pi)^{4}},
$$

$$
\begin{aligned}
U\left(\boldsymbol{p}, \boldsymbol{p}^{\prime}\right)= & V\left(\boldsymbol{p}, \boldsymbol{p}^{\prime}\right)+i \int V\left(\boldsymbol{p}, \boldsymbol{p}^{\prime \prime}\right) G_{\downarrow}^{N}\left(-p^{\prime \prime}\right) G_{\uparrow}^{N}\left(p^{\prime \prime}\right) \\
& \times U\left(\boldsymbol{p}^{\prime \prime}, \boldsymbol{p}^{\prime}\right) \theta\left(\left|\boldsymbol{p}^{\prime \prime}\right|-\Lambda\right) \frac{d^{4} p^{\prime \prime}}{(2 \pi)^{4}}
\end{aligned}
$$

In the second equation the full propagator $G_{\uparrow}(p)$ was replaced by its counterpart in the unpaired state, $G_{\uparrow}^{N}(p)$. By construction, the on-shell integration is carried over momenta much larger than the Fermi momentum for which the quasiparticle spectrum is unaffected by the pairing gap. Thus Eq. (17) decouples from Eq. (16), while the integration in Eq. (16) is now constrained to the vicinity of the Fermi surface. This permits us to simplify the problem by expanding the pairing interaction in spherical harmonics with respect to the angle formed by the momenta lying on the Fermi surface(s) and, thus, to reduce the pairing interaction to a function of a single angle. While this is useful for finite-range interactions, in the case of zero-range interactions the potential $V\left(\boldsymbol{p}, \boldsymbol{p}^{\prime}\right)$ needs to be eliminated from Eq. (17) in favor of the scattering $T$ matrix, which obeys the integral equation

$$
\begin{aligned}
T\left(\boldsymbol{p}, \boldsymbol{p}^{\prime}\right)= & V\left(\boldsymbol{p}, \boldsymbol{p}^{\prime}\right)+i \int_{0}^{\infty} V\left(\boldsymbol{p}, \boldsymbol{p}^{\prime \prime}\right) G_{\downarrow}^{N}\left(-p^{\prime \prime}\right) \\
& \times G_{\uparrow}^{N}\left(p^{\prime \prime}\right) T\left(\boldsymbol{p}^{\prime \prime}, \boldsymbol{p}^{\prime}\right) \frac{d^{4} p^{\prime \prime}}{(2 \pi)^{4}} .
\end{aligned}
$$

Combining Eqs. (17) and (18) one finds a regular integral equation defining the pairing force 


$$
\begin{aligned}
U\left(\boldsymbol{p}, \boldsymbol{p}^{\prime}\right)= & T\left(\boldsymbol{p}, \boldsymbol{p}^{\prime}\right)-i \int_{0}^{\Lambda} U\left(\boldsymbol{p}, \boldsymbol{p}^{\prime \prime}\right) G_{\downarrow}^{N}\left(-p^{\prime \prime}\right) \\
& \times G_{\uparrow}^{N}\left(p^{\prime \prime}\right) T\left(\boldsymbol{p}^{\prime \prime}, \boldsymbol{p}^{\prime}\right) \frac{d^{4} p^{\prime \prime}}{(2 \pi)^{4}} .
\end{aligned}
$$

In the dilute limit of interest, partial waves higher than the $s$ wave can be neglected, and the interaction is solely determined by the $s$-wave scattering length $a_{S}<0$, as $T\left(\boldsymbol{p}, \boldsymbol{p}^{\prime}\right)$ $=T_{0}=4 \pi\left|a_{S}\right| / \mathrm{m}$. The solution of Eq. (17) with this interaction is straightforward, and one obtains for $U\left(\boldsymbol{p}, \boldsymbol{p}^{\prime}\right)=U_{0}$

$$
U_{0}=T_{0}\left[1-\int G_{\downarrow}^{N}(-p) G_{\uparrow}^{N}(p) \theta(\Lambda-|\boldsymbol{p}|) \frac{d^{4} p}{(2 \pi)^{4}}\right]^{-1} .
$$

For the zero-range interaction above the gap equation takes the form

$$
\begin{aligned}
\Delta^{\dagger}(\boldsymbol{p}) & =U_{0} \int \operatorname{Im} F^{\dagger}\left(\boldsymbol{p}^{\prime}, \omega^{\prime}\right) f\left(\omega^{\prime}\right) \theta\left(\Lambda-\left|\boldsymbol{p}^{\prime}\right|\right) \frac{d^{3} p^{\prime} d \omega^{\prime}}{(2 \pi)^{4}} \\
& =\frac{U_{0}}{2} \int_{0}^{\Lambda} \frac{\Delta}{\sqrt{E_{S}^{2}+\Delta^{2}}}\left\langle f\left(\omega_{1}\right)-f\left(\omega_{2}\right)\right\rangle \frac{p^{\prime 2} d p^{\prime}}{(2 \pi)^{2}},
\end{aligned}
$$

where $\langle\cdots\rangle$ stands for the angle average and the second line follows in the quasiparticle approximation [i.e., by retaining only the pole part of the propagator $\left.F^{\dagger}(p)\right]$. The densities of the species are given by

$$
\begin{aligned}
\rho_{\uparrow / \downarrow} & =\int \operatorname{Im} G_{\uparrow \uparrow / \downarrow \downarrow}\left(\boldsymbol{p}^{\prime}, \omega\right) f(\omega) \frac{d^{3} p^{\prime} d \omega^{\prime}}{(2 \pi)^{4}} \\
& =\int\left\langle u\left(\boldsymbol{p}^{\prime}\right)^{2}\left[f\left(\omega_{\uparrow / \downarrow}\right)-f\left(-\omega_{\downarrow / \uparrow}\right)\right]+f\left(-\omega_{\downarrow / \uparrow}\right)\right\rangle \frac{p^{\prime 2} d p^{\prime}}{(2 \pi)^{2}},
\end{aligned}
$$

where $u(\boldsymbol{p})^{2}=1 / 2+E_{S} /\left(2 \sqrt{E_{S}^{2}+\Delta^{2}}\right)$ is the familiar Bogolyubov amplitude and the second line of Eq. (22) is obtained in the quasiparticle approximation. The normal selfenergy in the $T$-matrix (ladder) approximation is defined as

$$
\Sigma_{\sigma}(\boldsymbol{p})=i \sum_{\sigma^{\prime}} \int T_{\sigma \sigma^{\prime}}\left(\boldsymbol{p}, \boldsymbol{p}^{\prime} ; \varepsilon_{\boldsymbol{p}}+\omega^{\prime}\right) G_{\sigma^{\prime}}\left(p^{\prime}\right) \frac{d^{4} p^{\prime}}{(2 \pi)^{4}},
$$

where the dependence of the self-energy on the center-ofmass momentum is suppressed. If the $T$ matrix is approximated as above by a real constant $T_{0}$ which accounts for interactions between different species, the normal self-energy is momentum independent, purely real, and is given by

$$
\Sigma_{\uparrow / \downarrow}=T_{0} \rho_{\downarrow / \uparrow} .
$$

This constant shift in quasiparticle energy can be absorbed in the chemical potential by defining $\mu_{\uparrow / \downarrow}^{*}=\mu_{\uparrow / \downarrow}-\Sigma_{\downarrow / \uparrow}$. It is straightforward to extended this result to the case where the $T$ matrix contains components which act among the same species or includes contributions from partial waves with $L$ $>0$. Equations (20)-(22) and (24) are the fundamental equations of the mean-field theory for systems with unequal spin pairing that are interacting via zero-range forces. They include implicitly the effects of a finite momentum of Cooper pairs and the topology of the Fermi surfaces to which we shall turn in the following section.

\section{FINITE PAIR MOMENTUM AND DEFORMED FERMI SURFACES}

While the BCS ground state assumes that fermions bound in a Cooper pair have equal and opposite momenta (and spins), for fermionic systems with an unequal number of spin-up and -down particles this is not always true. Larkin and Ovchinnikov [39] and independently Fulde and Ferrell [40] observed that pairing is possible among pairs which have a finite total momentum with respect to some fixed reference frame. The finite momentum $\boldsymbol{P}$ changes the quasiparticle spectrum of the paired state. To see this note that the auxiliary propagator (9) written in the center-of-mass frame reads

$$
G_{\uparrow / \downarrow}^{N}(p, \boldsymbol{P})=\left[\omega-\frac{1}{2 m}\left(\frac{\boldsymbol{P}}{2} \pm \boldsymbol{p}\right)^{2}-\mu_{\uparrow / \downarrow}^{*}+i \eta\right]^{-1},
$$

and, therefore, the symmetric and antisymmetric (under time-reversal) parts of the quasiparticle spectrum are

$$
\begin{gathered}
E_{S}=\frac{1}{2}\left(\frac{P^{2}+4 p^{2}}{4 m}-\mu_{\uparrow}^{*}-\mu_{\downarrow}^{*}\right), \\
E_{A}=\frac{1}{2}\left(\frac{\boldsymbol{P} \cdot \boldsymbol{p}}{m}-\mu_{\uparrow}^{*}+\mu_{\downarrow}^{*}\right) .
\end{gathered}
$$

The results of Sec. III remain valid with the above redefinitions of $E_{S}$ and $E_{A}$. Note that the quantities of interest, in particular the gap, now depend parametrically on the total momentum. As a consequence, the twofold splitting of the spectrum (14) does not vanish in the limit of an equal number of spin-up and -down particles (i.e., when $\mu_{\uparrow}^{*}=\mu_{\downarrow}^{*}$ ). While such a state lowers the energy of the system with respect to the unpaired state, it is still unstable with respect to the ordinary BCS ground state.

We now turn to the deformations of the Fermi surfaces. The two Fermi surfaces for spin-up and -down particles are defined in momentum space by the equations $\varepsilon_{\uparrow / \downarrow}=\epsilon_{P / 2 \pm p}$ $-\mu_{\uparrow / \downarrow}^{*}=0$. When the states are filled isotropically within a sphere, the chemical potentials are related to the Fermi momentum $p_{F, \sigma}$ as $\mu_{\sigma}^{*}=p_{F, \sigma}^{2} / 2 m$ (for the sake of argument we assume here that the temperature is zero). To describe the deformations of Fermi surfaces from their spherical shape we expand the quasiparticle spectrum in spherical harmonics $\varepsilon_{\sigma}=\Sigma_{l} \varepsilon_{l \sigma} P_{l}(x)$, where $x$ is the cosine of the angle formed by the particle momentum and a randomly chosen symmetrybreaking axis and $P_{l}(x)$ are the Legendre polynomials. The $l=1$ terms break the translational symmetry by shifting the Fermi surfaces without deforming them; these terms are ignored below. Truncating the expansion at second order ( $l$ $=2$ ), we rewrite the spectrum in a form equivalent to the above one [38]:

$$
\varepsilon_{\uparrow / \downarrow}=\epsilon_{P \pm p}-\mu_{\uparrow / \downarrow}^{*}\left(1+\eta_{\uparrow / \downarrow} x^{2}\right),
$$

where the parameters $\eta_{\sigma}$ describe the quadrupole deformation of the Fermi surfaces. It is convenient to work with the 


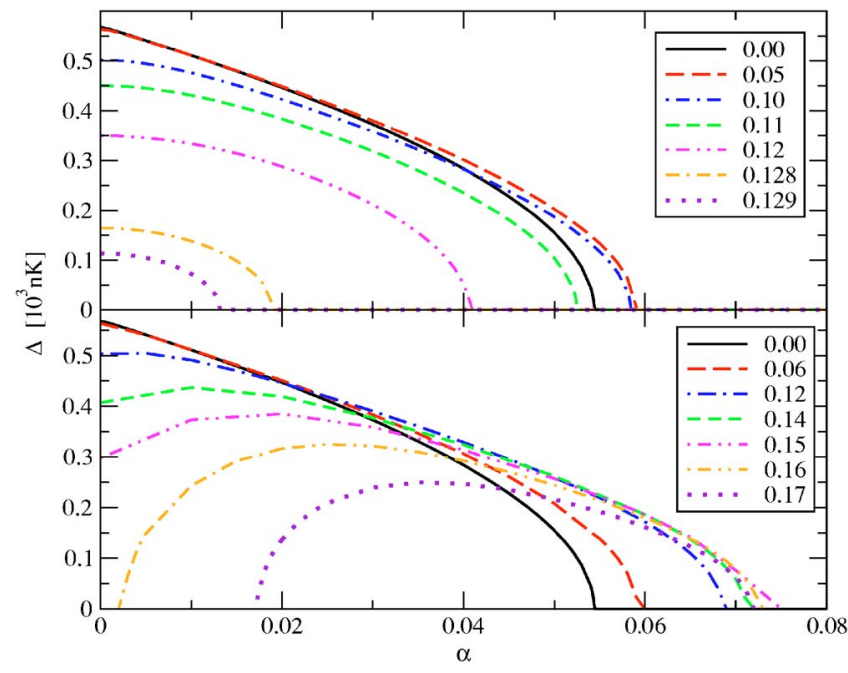

FIG. 1. (Color online) The dependence of the pairing gaps in the LOFF phase (upper panel) and the DFS phase (lower panel) on the asymmetry parameter for several values of the the total momentum $P / k_{F}$ and deformation parameter $\delta \epsilon$ which are indicated in the panels.

symmetrized $\Xi=\left(\eta_{\uparrow}+\eta_{\downarrow}\right) / 2$ and antisymmetrized $\delta \epsilon=\left(\eta_{\uparrow}\right.$ $\left.-\eta_{\downarrow}\right) / 2$ combinations of $\eta_{\uparrow, \downarrow}$. Below we shall assume $\Xi=0$ and consider two limiting cases $\delta \epsilon \neq 0$ and $\boldsymbol{P}=0$ [the phase referred as the Fermi surface superfluidity (DFS) phase] and $\delta \epsilon=0$ and $\boldsymbol{P} \neq 0$ (the plane-wave LOFF phase).

\section{RESULTS}

Consider a trap loaded with ${ }^{6} \mathrm{Li}$ atoms and assume that the net number of atoms in the trap is fixed while the system is maintained at constant temperature. Assume further that the number of atoms corresponds to a Fermi temperature $T_{F}$ $=\epsilon_{F} / k_{B}=942 \mathrm{nK}$, which in the uniform, symmetric case at $T=0$ would translate into a Fermi momentum of the system $k_{F} \approx 4.83 \times 10^{4} \mathrm{~cm}^{-1}$ and a density $\rho=3.8 \times 10^{12} \mathrm{~cm}^{-3}$. We shall work below at constant temperature $T=10 \mathrm{nk}\left(\ll T_{F}\right)$; i.e., the system is in a highly degenerate regime. (In the experiments of Ref. [53], such a Fermi temperature corresponds to about $4 \times 10^{5}$ atoms in a single hyperfine component.) Experiments control the partial densities of atoms in two different hyperfine states $|\uparrow\rangle=\left|F=3 / 2, m_{F}=3 / 2\right\rangle$ and $|\downarrow\rangle=|3 / 2,1 / 2\rangle$, e.g., by transferring atoms from one state to the other using $\sim 76 \mathrm{MHz}$ rf pulses [54]. Since the free-space triplet scattering length for ${ }^{6} \mathrm{Li}$ atoms in these hyperfine states is $a_{S}=-2160 a_{B}$, the system is in the weakly coupled regime $k_{F}\left|a_{S}\right| \ll \pi / 2$.

The pairing gaps of the LOFF and DFS phases computed from the coupled equations (21) and (22) are shown as a function of asymmetry parameter $\alpha=\left(\rho_{\uparrow}-\rho_{\downarrow}\right) /\left(\rho_{\uparrow}+\rho_{\downarrow}\right)$ for different values of the total momentum $P$ and deformation $\delta \epsilon$ in Fig. 1. Without loss of generality the density asymmetry is constrained to positive values; i.e., we assume $\rho_{\uparrow}>\rho_{\downarrow}$. The positive values of $\delta \epsilon$ correspond to a prolate (cigarlike) deformation of the majority and oblate (pancakelike) deformation of the minority population's Fermi spheres; we shall

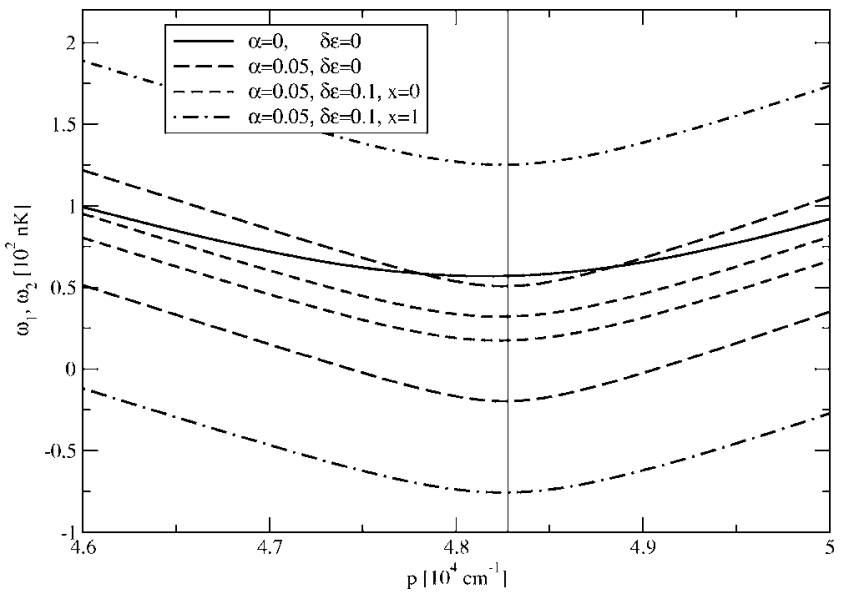

FIG. 2. The dependence of the quasiparticle spectra of two hyperfine states $\omega_{1}$ and $\omega_{2}$ on the momentum for $\alpha=0=\delta \epsilon$ (solid line), $\alpha=0.05$ and $\delta \epsilon=0$ (long-dashed lines), $\alpha=0.05, \delta \epsilon=0.1$, and $x=0$ (short-dashed lines), and $x=1$ (dash-dotted lines). The Fermi momentum $k_{F}=4.83 \times 10^{4} \mathrm{~cm}^{-1}$ is indicated by the vertical line.

confine ourselves to the case where $\delta \epsilon \geqslant 0$ since we have checked that it is the one corresponding to the largest value of the pairing energy.

To elucidate the dominance of the phases with brokenspace symmetries over the asymmetric BCS state consider the modifications in the single-particle spectra implied by these phases. In the asymmetric BCS state, the antisymmetric part of the quasiparticle spectrum (14), $E_{A}$, acts in the gap equation (21) to reduce the phase-space coherence between the quasiparticles that pair (when $E_{A}=0$ the BCS limit is recovered with equal occupations for both particles and perfectly matching Fermi surfaces). This blocking effect is responsible for the reduction of the gap with increasing asymmetry and its disappearance above $\alpha \simeq 0.055$.

When the pairs move with a finite total momentum or the Fermi surfaces are deformed, the antisymmetric part of the spectrum, $E_{A}$, is modulated with the cosine of the polar angle $x$ (in the frame where the $z$ axis is along the symmetrybreaking axis). In the case of the plane-wave LOFF phase $E_{A} \propto x$ while in the DFS phase $E_{A} \propto x^{2}$. This variation acts to restore the phase-space coherence for some values of $x$ at the cost of even lesser (than in the BCS phase) coherence for the remaining values. The effect can be seen explicitly in Fig. 2 which compares the quasiparticle spectra $\omega_{1}$ and $\omega_{2}$ in the DFS phase for combinations of $\alpha$ and $\delta \epsilon$ for two orthogonal directions with the BCS result for the symmetric case (black). Along the symmetry-breaking axis the energy separation between the quasiparticle spectra is considerably smaller than in the asymmetric BCS state; in the orthogonal direction, the opposite is the case. Compared to the asymmetric BCS state the phase-space overlap between pairs is increased in the first case and decreased in the second. The net result, displayed in Fig. 3, is the increase in the value of the critical asymmetry $\alpha_{c}$ at which the superfluidity vanishes. At large asymmetries the DFS phase exhibits the reentrance effect: the pairing exists only for the deformed state between the lower $\left(\alpha_{\mathrm{cr} 1}\right)$ and upper $\left(\alpha_{\mathrm{cr} 2}\right)$ critical deformations; see Fig. 3. This figure also shows the largest possible asymmetry 


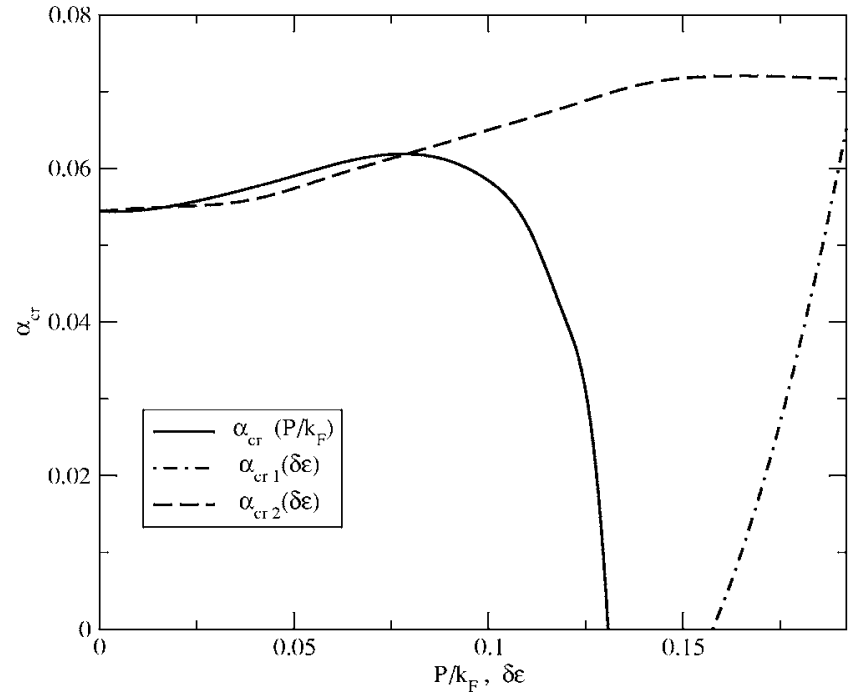

FIG. 3. The dependence of the critical asymmetry $\alpha_{c}$ of the transition from the superfluid to the normal state on the total momentum in the LOFF phase and the deformation parameter in the DFS phase. The model parameters are as in Fig. 1.

supported by the LOFF for a given total momentum $P$; this asymmetry is maximal at $P / k_{F} \sim 0.075$.

An important feature of the spectrum of the asymmetric BCS state $(\alpha \neq 0, \delta \epsilon=0=P)$ is its gapless nature [55-59]i.e., the existence of nodes for one (or both) branches of the spectra (cf. the gapped BCS spectrum also shown in Fig. 2). Gapless excitations affect the dynamical properties of the superfluid state such as the transport and collective modes and lead to a number of peculiarities in the thermodynamics of this state. This feature clearly remains intact for the phases with broken-space symmetries. As seen in Fig. 2 the spectrum of the DFS phase covers a range bounded by the curves with $x=0$ and $x=1$ and features nodes at which the quasiparticles can be excited by an infinitely small external perturbation. The macrophysical manifestations of the LOFF and DFS phases such as the response to density perturbations or electromagnetic probes and the thermodynamic functions (heat capacity, etc.) would differ from the ordinary BCS phase due to the nodes and anisotropy of their spectrum. Such an anisotropy can be used to discriminate phases with broken-space symmetries in the time-of-flight experiments (see Sec. VI). Moreover, the phases with broken-space symmetries feature a larger number of Goldstone modes than the asymmetric BCS phase because of the breaking of additional global space symmetries.

Which phase is the true ground state at a give density asymmetry is decided by a comparison of the free-energy difference $\Delta F=F_{S}-F_{N}$ of these phases which are displayed in Fig. 4. The LOFF phase is preferred to the normal and homogeneous BCS phases in a narrow window of asymmetries, $0.04 \leqslant \alpha \leqslant 0.057$, and for the total momentum of the pairs, $P / k_{F} \sim 0.05$. This is consistent with the results obtained in the scheme where the density asymmetry is described in terms of the difference in the chemical potentials of the species $\delta \mu$ [the critical value for the BCS phase is $\delta \mu_{c}^{B C S}=0.707 \Delta(0)$, while for the LOFF phase $\delta \mu_{c}^{L O F F}$

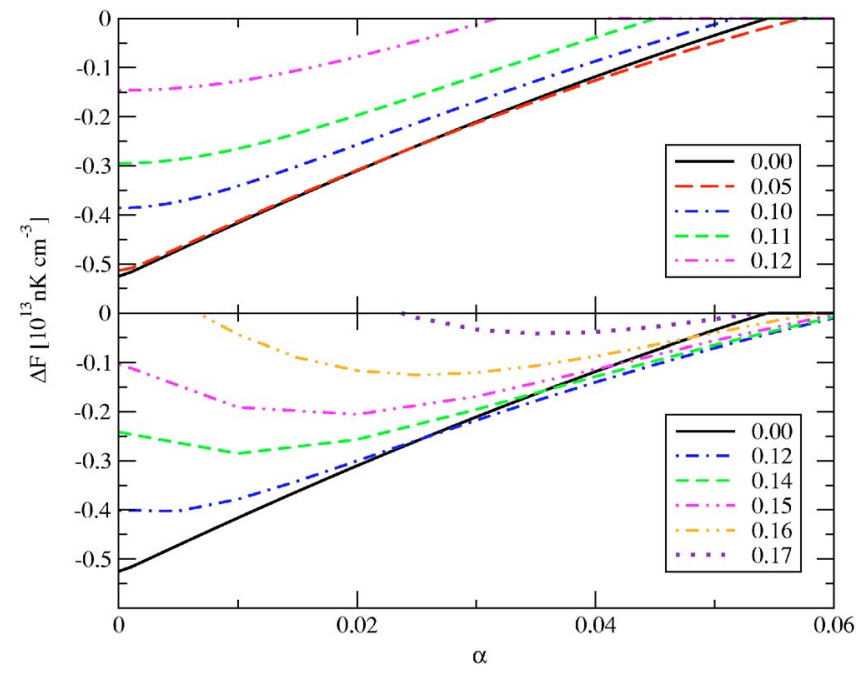

FIG. 4. (Color online) The dependence of the free energy of the LOFF (upper panel) and the plane-wave DFS phase (lower panel) on the asymmetry parameter for several values of the deformation parameter $\delta \epsilon$ and the total momentum $P / k_{F}$ which are indicated in the panels.

$=0.755 \Delta(0)$, where $\Delta(0) \equiv \Delta(\delta \mu=0)][39,40]$. Note that while there is a nontrivial solution to the gap equation for $P / k_{F} \geqslant 0.01$, the gain in the pairing energy is less than the loss in kinetic energy due to the motion of the condensate and the net energy of the LOFF phase is larger than that of the asymmetric BCS phase. However, the pairing energy of the LOFF phase can be increased by choosing a more complex form of the order parameter-e.g., by keeping a larger number of terms in the expansion of the order parameter in the Fourier series.

The DFS phase is the ground state of the system (i.e., it has a lower free energy than the normal, BCS, and LOFF phases) in a wider range of asymmetries, $0.03 \leqslant \alpha \leqslant 0.06$, for the deformation parameters in the range $0.12 \leqslant \delta \epsilon \leqslant 0.16$. For even larger deformations the gain in the pairing energy does not compensate the loss in the kinetic energy due to the stretching of the Fermi surface into ellipsoidal form. Note that the free energy is also affected by the reentrance effect (i.e., restoration of pairing correlations as the asymmetry is increased). However, while beyond the reentrance point the DFS phase becomes preferable to the unpaired state, its free energy is still larger than in the homogenous asymmetric BCS state. Only at the large asymmetries quoted above does it become the ground state of the system.

To summarize, the coherence is restored and the strength of pair correlations is increased in the LOFF phase due to the finite momentum of the Cooper pairs. In the DFS phase the same is achieve by stretching the spherical Fermi surfaces into ellipsoids. The fundamental difference between these phases is that the translational symmetry remains intact for the DFS phase, which breaks only the rotational symmetry, while the LOFF phase breaks both symmetries. Quantitatively, the maximal value of the gap and the absolute value of the ground-state free energy is larger in the DFS phase than in the LOFF phase for asymmetries larger than $\alpha \simeq 0.04$. For these asymmetries both phases are favorable over the homo- 


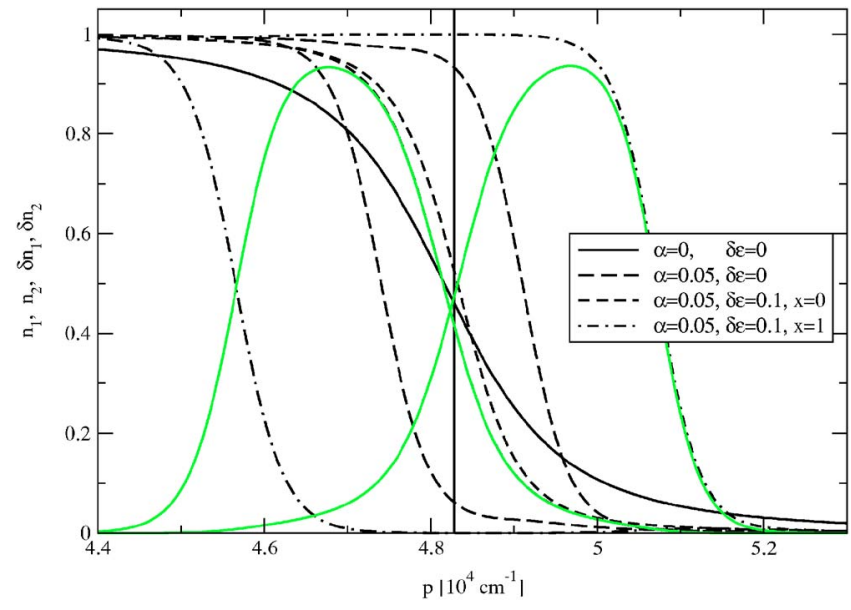

FIG. 5. (Color online) The dependence of the occupation probabilities of two hyperfine states on the momentum. The Fermi momentum $k_{F}=4.83 \times 10^{4} \mathrm{~cm}^{-1}$ is indicated by the vertical line. The labeling of the lines is as follows: $\alpha=0=\delta \epsilon$ (solid line), $\alpha=0.05$ and $\delta \epsilon=0$ (long-dashed lines), $\alpha=0.05, \delta \epsilon=0.1$, and $x=0$ (short-dashed lines), and $x=1$ (dash-dotted lines). The bell-shaped light curves show the anisotropy-the difference between the $x=1$ and $x=0$ occupation numbers-for $\alpha=0.05, \delta \epsilon=0.1$. The remaining parameters are as in Fig. 1.

geneous BCS phase. However, one should keep in mind that the LOFF phase admits a variety of lattice forms and the plane-wave structure need not be the most favored one [46].

\section{DETECTING THE DFS PHASE IN EXPERIMENTS}

The large number of side effects in solid systems (such as the presence of the ionic lattice or defects) have precluded up to now a clear detection of a superfluid phase with brokenspace symmetries in these systems. The situation can be much more favorable in ultracold gases due to the high control over the experimental conditions. Recently, Mizushima et al. proposed a way to detect the LOFF phase in these systems by imaging the density profiles by local spin measurements [44]. Below we propose a simple method to detect the DFS phase in a Fermi system with rotational symmetrye.g., a system which is either homogeneous or in a spherical trap. Indeed, experimental evidence for the phases with broken-space symmetries can be obtained from studies of their momentum distributions which, unlike in the homogeneous phase, must be anisotropic in space. Figure 5 shows the occupation numbers in the BCS, the asymmetric BCS, and the DFS phases; varying the cosine of the polar angle $x$ covers a range of occupation probabilities which includes the undeformed asymmetric state. The bell-shaped curves show the angular polarization of the occupation numbers in the DFS phase defined as $\delta n_{\sigma}=\left|n_{\sigma}(x=1)-n_{\sigma}(x=0)\right|$. The maximal anisotropy in the occupation probabilities of particles along and orthogonal to the symmetry-breaking axis is about $90 \%$. Thus, a direct way to detect the DFS phase is the measurement of the anisotropy in the momentum distribution of the trapped atoms. Such a measurement can be realized by the time-of-flight technique $[1-7,26]$. This method uses the fact that after releasing the trap, the atoms fly out freely and an image of their spatial distribution taken after some time of flight provides information on their momentum distribution when confined inside the trap. Assuming that the system was in the deformed superfluid state one would detect a mean momentum of particles of type 1 (majority) in the direction of symmetry breaking by about $90 \%$ larger than that of particles of type 2 (minority) in the same direction. Therefore, the presence of this anisotropy in the detected momentum distributions would be evidence for a deformed superfluid state being the ground state of the system, as deformation alone (i.e., without pairing) would not lower the energy so as to produce a deformed nonsuperfluid ground state. Note that this argument is equally valid for a homogeneous system or for an atomic gas in a spherical trap, where no preferred direction is introduced by the trapping potential. For a nonspherical trap, the momentum distributions of both species are expected to be deformed in the same way; therefore, the detection of an anisotropy in momentum distributions superimposed on the deformation induced by the deformed trap could be a signature of the DFS phase. Case studies for certain forms of deformed trapped (e.g., in the local density approximation) would be necessary to quantify the effect of combined deformation due to the trap and pairing.

The direction of spontaneous symmetry breaking (in $k$ space and, therefore, also in real space) is chosen by the system randomly and needs to be located in an experiment to obtain maximum anisotropy. A clear distinction between the DFS and LOFF phases can be achieved in time-of-flight experiments, since the latter predicts periodic momentum distributions, independent of the detailed spatial structure of the phase that nucleates in the ground state.

\section{ACKNOWLEDGMENTS}

This work was supported by the DGICYT Grant Nos. BFM2002-01868 and HA2001-0023 [Acciones Integradas] (Spain), the Deutsche Akademische Austausch Dienst and the Sonderforschungsberich 381 of the Deutsche Forschungsgemeinschaft (Germany), and the Generalitat de Catalunya (J.M.-P.)
[1] B. DeMarco and D. S. Jin, Science 285, 1703 (1999).

[2] K. M. O'Hara, S. L. Hemmer, M. E. Gehm, S. R. Granade, and J. E. Thomas, Science 13, 2179 (2002).

[3] C. A. Regal, C. Ticknor, J. L. Bohn, and D. S. Jin, Phys. Rev.
Lett. 90, 053201 (2003).

[4] K. E. Strecker, G. B. Partridge, and R. G. Hulet, Phys. Rev. Lett. 91, 080406 (2003).

[5] M. W. Zwierlein, C. A. Stan, C. H. Schunck, S. M. F. Raupach, 
S. Gupta, Z. Hadzibabic, and W. Ketterle, Phys. Rev. Lett. 91, 250401 (2003).

[6] M. Greiner, C. A. Regal, and D. S. Jin, Nature (London) 426, 537 (2003).

[7] C. A. Regal, M. Greiner, and D. S. Jin, Phys. Rev. Lett. 92, 040403 (2004).

[8] T. Bourdel, L. Khaykovich, J. Cubizolles, J. Zhang, F. Chevy, M. Teichmann, L. Tarruell, S. J. J. M. F. Kokkelmans, and C. Salomon, Phys. Rev. Lett. 93, 050401 (2004).

[9] H. T. C. Stoof, M. Houbiers, C. A. Sackett, and R. G. Hulet, Phys. Rev. Lett. 76, 10 (1996).

[10] M. Houbiers, R. Ferwerda, H. T. C. Stoof, W. I. McAlexander, C. A. Sackett, and R. G. Hulet, Phys. Rev. A 56, 4864 (1997).

[11] W. C. Stwalley, Phys. Rev. Lett. 37, 1628 (1976).

[12] E. Tiesinga, B. J. Verhaar, and H. T. C. Stoof, Phys. Rev. A 47, 4114 (1993).

[13] Ph. Courteille, R. S. Freeland, D. J. Heinzen, F. A. van Abeelen, and B. J. Verhaar, Phys. Rev. Lett. 81, 69 (1998).

[14] J. L. Bohn, Phys. Rev. A 61, 053409 (2000).

[15] M. Holland, S. J. J. M. F. Kokkelmans, M. L. Chiofalo, and R. Walser, Phys. Rev. Lett. 87, 120406 (2001).

[16] E. Timmermans, K. Furuya, P. W. Milonni, and A. K. Kerman, Phys. Lett. A 285, 228 (2001).

[17] H. Heiselberg, Phys. Rev. A 63, 043606 (2001).

[18] M. L. Chiofalo, S. J. J. M. F. Kokkelmans, J. N. Milstein, and M. J. Holland, Phys. Rev. Lett. 88, 090402 (2002).

[19] Y. Ohashi and A. Griffin, Phys. Rev. Lett. 89, 130402 (2002).

[20] J. Stajic, J. N. Milstein, Qijin Chen, M. L. Chiofalo, M. J. Holland, and K. Levin, Phys. Rev. A 69, 063610 (2004).

[21] R. Combescot, Phys. Rev. Lett. 91, 120401 (2003).

[22] G. M. Falco and H. T. C. Stoof, Phys. Rev. Lett. 92, 130401 (2004).

[23] J. Kinnunen, M. Rodriguez, and P. Törma, Phys. Rev. Lett. 92, 230403 (2004).

[24] G. M. Bruun and C. J. Pethick, Phys. Rev. Lett. 92, 140404 (2004).

[25] J. Zhang, E. G. M. van Kempen, T. Bourdel, L. Khaykovich, J. Cubizolles, F. Chevy, M. Teichmann, L. Tarruell, S. J. J. M. F. Kokkelmans, and C. Salomon, Phys. Rev. A 70, 030702(R) (2004).

[26] C. A. Regal, M. Greiner, and D. S. Jin, Phys. Rev. Lett. 92, 040403 (2004).

[27] M. W. Zwierlein, C. A. Stan, C. H. Schunck, S. M. F. Raupach, A. J. Kerman, and W. Ketterle, Phys. Rev. Lett. 92, 120403 (2004).

[28] J. Kinast, S. L. Hemmer, M. E. Gehm, A. Turlapov, and J. E. Thomas, Phys. Rev. Lett. 92, 150402 (2004).

[29] F. Schreck, G. Ferrari, K. L. Corwin, J. Cubizolles, L. Khaykovich, M.-O. Mewes, and C. Salomon, Phys. Rev. A 64, 011402(R) (2001).

[30] T. Bourdel, J. Cubizolles, L. Khaykovich, K. M. F. Magalhaes, S. J. J. M. F. Kokkelmans, G. V. Shlyapnikov, and C. Salomon, Phys. Rev. Lett. 91, 020402 (2003).

[31] M. A. Baranov and D. S. Petrov, Phys. Rev. A 62, 041601(R) (2004).
[32] S. Stringari, Europhys. Lett. 65, 749 (2004).

[33] C. Chin, M. Bartenstein, A. Altmeyer, S. Riedl, S. Jochim, J. Hecker Denschlag, and R. Grimm, Science 305, 1128 (2004).

[34] At the relevant temperatures (in the nanokelvin range) the pairing in $L \neq 0$ orbital angular momentum is less favored, unless one deals with the pairing among indistinguishable particles where relative $s$-wave pairing is forbidden by the Pauli principle.

[35] Qijin Chen, Jelena Stajic, Shina Tan, and K. Levin, Phys. Rep. 412, 1 (2005).

[36] J. Mur-Petit, A. Polls, and H.-J. Schulze, Phys. Lett. A 290, 317 (2001).

[37] U. Lombardo, P. Nozières, P. Schuck, H.-J. Schulze, and A. Sedrakian, Phys. Rev. C 64, 064314 (2001).

[38] H. Müther and A. Sedrakian, Phys. Rev. Lett. 88, 252503 (2002); Phys. Rev. C 67, 015802 (2003).

[39] A. I. Larkin and Y. N. Ovchinnikov, Zh. Eksp. Teor. Fiz. 47, 1136 (1964); [Sov. Phys. JETP 20, 762 (1965)].

[40] P. Fulde and R. A. Ferrell, Phys. Rev. 135, A550 (1964).

[41] R. Combescot, Europhys. Lett. 55, 15 (2001).

[42] R. Combescot and C. Mora, Eur. Phys. J. B 28, 397 (2002).

[43] C. Mora and R. Combescot, Physica B 329, 1435 (2003).

[44] T. Mizushima, K. Machida, and M. Ichioka, Phys. Rev. Lett. 94, 060404 (2005).

[45] P. Castorina, M. Grasso, M. Oertel, M. Urban, and D. Zappala, e-print cond-mat/0504598.

[46] M. Alford, J. A. Bowers, and K. Rajagopal, Phys. Rev. D 63, 074016 (2001); A. Sedrakian, Phys. Rev. C 63,025801 (2001); J. A. Bowers and K. Rajagopal, Phys. Rev. D 66, 065002 (2002); R. Casalbuoni and G. Nardulli, Rev. Mod. Phys. 76, 263 (2004).

[47] A. Sedrakian, J. Mur-Petit, A. Polls, and H. Müther, e-print cond-mat/0404577.

[48] P. F. Bedaque, Nucl. Phys. A 697, 569 (2002).

[49] P. F. Bedaque, H. Caldas, and G. Rupak, Phys. Rev. Lett. 91, 247002 (2003).

[50] H. Caldas, Phys. Rev. A 69, 063602 (2004).

[51] A. A. Abrikosov, L. P. Gor'kov, and I. E. Dzyaloshinski, Methods of Quantum Field Theory in Statistical Physics (Dover, New York, 1963).

[52] J. W. Serene and D. Reiner, Phys. Rep. 101, 221 (1983).

[53] Z. Hadzibabic, C. A. Stan, K. Dieckmann, S. Gupta, M. W. Zwierlein, A. Gorlitz, and W. Ketterle, Phys. Rev. Lett. 88, 160401 (2002).

[54] Z. Hadzibabic, Ph.D. thesis, MIT (2003), Chap. 5 [available at http://rleweb.mit.edu/cua_pub/ketterle_group/Theses/ Zoran_Thesis. pdf].

[55] G. Sarma, J. Phys. Chem. Solids 24, 1029 (1963).

[56] A. Sedrakian and U. Lombardo, Phys. Rev. Lett. 84, 602 (2000); A. Sedrakian, T. Alm, and U. Lombardo, Phys. Rev. C 55, R582 (1997).

[57] W. V. Liu and F. Wilczek, Phys. Rev. Lett. 90, 047002 (2003).

[58] I. Shovkovy and M. Huang, Phys. Lett. B 564, 205 (2003); M. Huang and I. Shovkovy, Nucl. Phys. A 729, 835 (2003).

[59] S.-T. Wu and S. Yip, Phys. Rev. A 67, 053603 (2003). 